\title{
Hairy rotating black string in the Einstein-Maxwell-Higgs system
}

\author{
M. H. Dehghani ${ }^{1,2 *}$ and A. Khodam-Mohammadi ${ }^{1}$ \\ 1. Physics Department and Biruni Observatory, \\ College of Sciences, Shiraz University, Shiraz 71454, Iran \\ 2. Institute for Studies in Theoretical Physics and \\ Mathematics (IPM), P.O. Box 19395-5531, Tehran, Iran
}

\begin{abstract}
We show numerically that the Abelian Higgs field equations in the background of a four-dimensional rotating charged black string have vortex solutions. These solutions which have axial symmetry show that the rotating black string can support the Abelian Higgs field as hair. We find that one encounters with an electric field coupled to the Higgs scalar field for the case of rotating black string. This electric field is due to an electric charge per unit length, which increases as the rotation parameter becomes larger. We also find that the vortex thickness decreases as the rotation parameter grows up. Finally we consider the self-gravity of the Abelian Higgs field and show that the effect of the vortex is to induce a deficit angle in the metric under consideration which decreases as the rotation parameter increases.
\end{abstract}

\section{INTRODUCTION}

The classical no hair conjecture, first proposed by Ruffini and Wheeler states that the only allowed characteristics of stationary black holes are those associated with the Gauss law, such as mass, angular momentum and electromagnetic charge 1]. Thus, for example, it follows that black holes cannot support external scalar fields, since there is no Gauss law for scalars. This fact is verified in [2] for a scalar field minimally coupled to gravity and in [3] for non-minimally coupled scalar field in asymptotic flat spacetimes. However, recently, it is shown that there exist scalar hairy black hole if one uses a scalar potential with regions violating the weak energy condition [4]. In recent year, we are faced with new black hole solutions in special gauge theories that Einstein's equation is coupled with

\footnotetext{
*Electronic address: mhd@shirazu.ac.ir
} 
some gauge fields, and therefore this conjecture needs more investigation. For example the Einstein-Yang-Mills black hole possess a short ranged external non Abelian gauge field and is not uniquely specified by their mass, angular momentum and electromagnetic charge [5]. Although this solution is unstable [6], it opened up new possibility that the black holes may have various matter hairs. The global monopole field is an example of stable hair for asymptotically flat black hole 7]. Also explicit calculations have been carried out which verify the existence of a long range Nielson-Olesen vortex solution as a stable hair for a static black hole solution of Einstein's equation in four dimensions [8]. For the extreme black hole of Einstein-Maxwell gravity, it has been shown that flux expulsion occurs for thick strings (thick with respect to the radius of horizon), while flux penetration occurs for thin strings 9, 10, 11, 12]. Of course one may note that these situations fall out side of the scope of the classical no hair theorem due to the non trivial topology of the string configuration.

It has recently been shown that these ideas can be extended to the case of anti de sitter (AdS) and de Sitter (dS) spacetimes. For asymptotically AdS spacetimes, it has been shown that conformally coupled scalar field can be painted as hair [13]. Another asymptotically AdS hairy black hole solution has been investigated in Ref. [14]. Also it was shown that there exist a solution to the $S U(2)$ Einstein-Yang-Mills equations which describes a stable YangMills hairy black hole, that is asymptotically AdS [15]. Although the idea of Nielson-Olesen vortices has been first introduced in flat spacetimes [16], but recently it has been extended to (A)dS spacetimes [17, 18]. The existence of long range Nielson-Olesen vortex as hair for asymptotically AdS black holes has been investigated in Refs. [19, 20] for Schwarzschild-AdS black hole and charged black string.

The presence of a rotation parameter introduces significant additional difficulties for explicit calculations intended to investigate the existence of a long range Nielson-Olesen vortex solution as a hair for a stationary black hole solution [21]. In this paper we study the Abelian Higgs hair in a four dimensional rotating charged black string that is a stationary model for Einstein-Maxwell equations in cylindrical symmetry. Various features of this kind of solutions of Einstein-Maxwell equations have been considered [22, 23]. Here, we want to investigate the influence of rotation on the vortex solution of Einstein-Maxwell-Higgs equations. Since an analytic solution to the Abelian Higgs field equations appears to be intractable, we confirm by numerical calculations that the rotating charged black string can be dressed by Abelian Higgs field as hair. 
In Sec. III, we consider the Abelian Higgs field equations in the background of a rotating charged black string. Section $\amalg$ is devoted to the numerical solutions of the field equations for different value of the rotation parameter and winding number. In Sec. IV by studying the behavior of the Abelian Higgs field energy-momentum tensor, we find the effect of the vortex self-gravity on the rotating charged black string background. We append some closing remarks in Sec. $\nabla$

\section{FIELD EQUATIONS}

The Lagrangian of Einstein gravity in the presence of electromagnetic and Abelian Higgs fields is 9 ]

$$
\mathcal{L}=\mathcal{R}-2 \Lambda-F_{\mu \nu} F^{\mu \nu}+\mathcal{L}_{H},
$$

where $\Lambda=-3 / l^{2}$ is the negative cosmological constant and $F_{\mu \nu}=\partial_{\mu} A_{\nu}-\partial_{\nu} A_{\mu}$ is the field strength tensor associated with the electromagnetic field $A_{\mu}$. The first three terms in Eq. (11) are the Einstein-Maxwell volume terms with negative cosmological constant, and $\mathcal{L}_{H}$ is the Lagrangian for Abelian Higgs system minimally coupled to gravity given as

$$
\mathcal{L}_{H}=-\frac{1}{2}\left(\mathcal{D}_{\mu} \Phi\right)^{\dagger} \mathcal{D}^{\mu} \Phi-\frac{1}{16 \pi} \mathcal{F}_{\mu \nu} \mathcal{F}^{\mu \nu}-\xi\left(\Phi^{\dagger} \Phi-\eta^{2}\right)^{2} .
$$

The matter content of the Abelian Higgs system consists of the complex Higgs field, $\Phi$, and a $U(1)$ gauge field $B_{\mu}$ with strength $\mathcal{F}_{\mu \nu}=\partial_{\mu} B_{\nu}-\partial_{\nu} B_{\mu}$. Both the Higgs scalar and the gauge field become massive in the broken symmetry phase. They are coupled through the gauge covariant derivatives $\mathcal{D}_{\mu}=\nabla_{\mu}+i e B_{\mu}$, where $\nabla_{\mu}$ is the spacetime covariant derivative. We use units in which $8 \pi G=c=1$.

The fields in $\mathcal{L}_{H}$ will be treated as "test field", i.e., their energy momentum tensor is supposed to yield a negligible contribution to the source of the gravitational field. Notice that we have two different gauge fields, $F$ and $\mathcal{F}$, and each is treated in a different manner. It is only $\mathcal{F}$ that couples to the Higgs scalar field and is therefore subject to spontaneous symmetry breaking. The other gauge field, $F$, can be thought of as the free massless Maxwell field which apart from modifying the background geometry, its dynamic will be of little concern to us here. It is conventional to express the field equations in terms of real fields $X\left(x^{\mu}\right), \omega\left(x^{\mu}\right)$, and $P_{\mu}\left(x^{\nu}\right)$ defined as

$$
\Phi\left(x^{\mu}\right)=\eta X\left(x^{\mu}\right) e^{i \omega\left(x^{\mu}\right)},
$$




$$
B_{\mu}\left(x^{\nu}\right)=\frac{1}{e}\left[P_{\mu}\left(x^{\nu}\right)-\nabla_{\mu} \omega\left(x^{\mu}\right)\right]
$$

where $X$ and $P_{\mu}$ are the scalar Higgs field and massive vector boson, and $\omega$ is a gauge degree of freedom. In terms of these new variables, the Lagrangian, $\mathcal{L}_{H}$ and the field equations can be written as

$$
\begin{aligned}
& \mathcal{L}_{H}\left(X, P_{\mu}\right)=-\frac{\eta^{2}}{2}\left(\nabla_{\mu} X \nabla^{\mu} X+X^{2} P_{\mu} P^{\mu}\right)-\frac{1}{16 \pi e^{2}} F_{\mu \nu}^{\prime} F^{\prime \mu \nu}-\xi \eta^{4}\left(X^{2}-1\right), \\
& \nabla_{\mu} \nabla^{\mu} X-X P_{\mu} P^{\mu}-4 \xi \eta^{2} X\left(X^{2}-1\right)=0 \\
& \nabla_{\mu} F^{\prime \mu \nu}-4 \pi e^{2} \eta^{2} P^{\nu} X^{2}=0, \\
& G_{\mu \nu}-\frac{3}{l^{2}} g_{\mu \nu}=T_{\mu \nu}^{e m}+\mathcal{T}_{\mu \nu}^{\text {Higgs }},
\end{aligned}
$$

where $F^{\mu \nu}=\nabla^{\mu} P^{\nu}-\nabla^{\nu} P^{\mu}$ is the field strength of the corresponding gauge field $P^{\mu}$, and $T_{\mu \nu}^{e m}$ and $\mathcal{T}_{\mu \nu}^{\text {Higgs }}$ are the stress energy tensors of the electromagnetic and Higgs fields given by

$$
\begin{aligned}
& T_{\mu \nu}^{e m}=2 F_{\mu}^{\sigma} F_{\sigma \nu}-\frac{1}{2} F^{2} g_{\mu \nu}, \\
& \mathcal{T}_{\mu \nu}^{\text {Higgs }}=\eta^{2} \nabla_{\mu} X \nabla_{\nu} X+\eta^{2} X^{2} P_{\mu} P_{\nu}+\frac{1}{4 \pi e^{2}} \mathcal{F}_{\mu \sigma} \mathcal{F}_{v}^{\sigma}+g_{\mu \nu} \mathcal{L}_{H} .
\end{aligned}
$$

Note that the real field $\omega$ is not itself a physical quantity. Superficially it appears not to contain any physical information. However, if $\omega$ is not single valued this is no longer the case, and the resultant solutions are referred to as vortex solutions [16]. In the presence of vortex, the requirement that the $\Phi$ field be single valued implies that the line integral of $\omega$ over any closed loop is $\pm 2 \pi N$, where $N$ is the winding number of the vortex. In this case the flux of the field $\mathcal{F}$ passing through such a closed loop is quantized with quanta $2 \pi / e$.

It must be noted that no exact solution are known for the coupled Einstein-Higgs equations (5)-(8) even in situations having both no electromagnetic charge and no horizon. Thus we should try to solve these coupled differential equations approximately. In the first order approximation, we solve Eqs. (15) and (17) in the background of charged rotating black string and then we will carry out numerical calculations to solve Eq. (8) . The metric of the charged rotating black string can be written as [22, 24]

$$
d s^{2}=-\Gamma(\Xi d t-a d \phi)^{2}+\frac{r^{2}}{l^{4}}\left(a d t-\Xi l^{2} d \phi\right)^{2}+\frac{d r^{2}}{\Gamma}+\frac{r^{2}}{l^{2}} d z^{2},
$$

where

$$
\Gamma=\frac{r^{2}}{l^{2}}-\frac{b l}{r}+\frac{\lambda^{2} l^{2}}{r^{2}}, \quad A_{\mu}=-\frac{\lambda l}{r}\left(\Xi \delta_{\mu}^{0}-a \delta_{\mu}^{\phi}\right), \quad \Xi=\sqrt{1+a^{2} / l^{2}} .
$$


$b$ and $\lambda$ are the constant parameters of the metric which are related to the mass and charge per unit length of the black string by $M=b / 4$ and $Q=\lambda / 2$, and $a$ is the rotation parameter. It is remarkable to note that the metric (111) reduces to AdS spacetime as $t$ goes to infinity in contrast to the Kerr-AdS metric, for which the surface at infinity is also rotating. The horizon of this black string has cylindrical symmetry that has two inner and outer horizon at $r_{-}$and $r_{+}$, provided that $b$ is greater than $b_{\text {crit }}$ given by

$$
b_{\text {crit }}=4 \times 3^{-3 / 4} \lambda^{3 / 2} .
$$

In the case that $b=b_{c r i t}$, we will have an extreme black string.

We seek a cylindrically symmetric solution for the Higgs field equations (5D) and (7D) in the background of a charged rotating black string. Thus, we assume that the fields $X$ and $P_{\mu}$ are functions of $r$. For the case of vanishing rotation parameter, one can choose the gauge field as $P_{\mu}(r)=(0,0, N p(r), 0)$. The field equations (5) and (17) reduce in that case to two equations for the two unknown functions $X(r)$ and $P(r)$. In the present case the field equations (5) and (7) reduce to three equations and one may therefore use the gauge choice

$$
P_{\mu}(r)=(S(r), 0, N P(r), 0) .
$$

The field equations (15) and (7) reduce to

$$
\begin{aligned}
& r^{2} \Gamma X^{\prime \prime}+\left(4 l^{-2} r^{3}-b l\right) X^{\prime}-4 r^{2} X\left(X^{2}-1\right)-X(a S+\Xi N P)^{2}- \\
& -r^{2} \Gamma^{-1} X\left(\Xi S+N a l^{-2} P\right)^{2}=0, \\
& N\left(r^{3} l^{2} \Gamma \Xi^{2}-r^{5} a^{2} l^{-2}\right) P^{\prime \prime}+N\left(2 r^{4}+b l^{3} r \Xi^{2}-2 \lambda^{2} l^{4} \Xi^{2}\right) P^{\prime}+\left(l^{2} \Xi^{2}-r^{2} a^{2} l^{-2} \Gamma^{-1}\right) \times \\
& N \alpha r^{3} X^{2} P+l^{2} a \Xi\left(\lambda^{2} l^{2}-b l r\right)\left(r S^{\prime \prime}-S^{\prime}+\alpha r X^{2} \Gamma^{-1} S\right)-a \Xi \lambda^{2} l^{4} S^{\prime}=0, \\
& r^{3}\left(\Xi^{2} r^{2}-\Gamma a^{2}\right) S^{\prime \prime}+\left[2 r^{4}-a^{2}\left(b l r-2 \lambda^{2} l^{2}\right)\right] S^{\prime}+\alpha X^{2} r^{3} \Gamma^{-1}\left(\Xi^{2} r^{2}-\Gamma a^{2}\right) S \\
& -N \Xi a\left[\left(r P^{\prime \prime}-P^{\prime}+\alpha r X^{2} \Gamma^{-1} P\right)\left(\lambda^{2} l^{2}-b l r\right)-\lambda^{2} l^{2} P^{\prime}\right]=0,
\end{aligned}
$$

where $\alpha=4 \pi e^{2} / \xi$ and the prime denotes a derivative with respect to $r$. It is worthwhile to mention that even in the pure flat or (anti) de Sitter spacetimes no exact analytic solutions are known for equations (5) and (7). For asymptotically AdS spacetimes, one of us have showed that the Abelian Higgs equations of motion in the background of charged black string spacetime have vortex solution [20]. Here we want to investigate the influence of rotation parameter on the vortex solutions. 


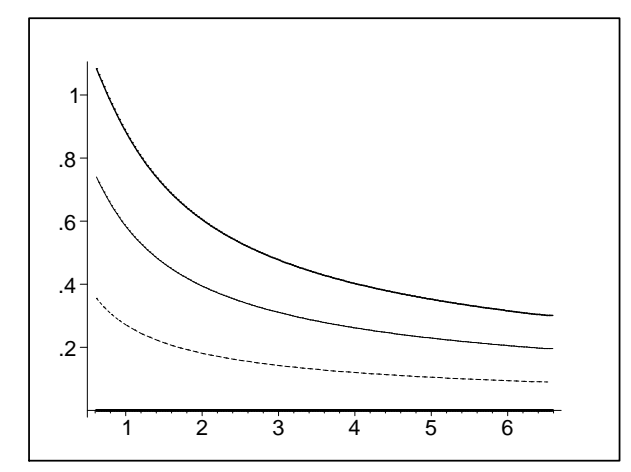

FIG. 1: $E_{\text {Higgs }} \times 10^{3}$ versus $r$ for $N=1, a=0$ (touch the horizontal axis), 0.25 (dotted), 0.5 (solid) and 0.7 (bold).

\section{NUMERICAL SOLUTIONS}

The main difference between this rotating black string and the non rotating case considered in Ref. [20] is the following. In the static case we had only two unknown functions $X(r)$ and $P(r)$, but here we encounter with three unknown functions $X(r), P(r)$ and $S(r)$. The latter function $S(r)$ which is the time component of $B_{\mu}$ creates a radial electric-type field, $F_{t r}$ that goes to zero as the rotation parameter goes to zero. It should be noted that even in the case of $b=\lambda=0$, no exact analytic solutions are known for Eqs. (15)-(17). So, here we seek the existence of vortex solutions for these coupled differential equations numerically. First, we must take appropriate boundary conditions. Since at a large distance from the horizon the metric (11) reduces to AdS spacetime, we demand that our solutions go to the solutions of the vortex equations in AdS spacetime given in 17]. This requires that we demand $(X \rightarrow 1, P \rightarrow 0)$ as $r$ goes to infinity and $(X=0, P=1)$ on the horizon. For consistency with the non-rotating case [20], we take $S=0$ on the horizon. Also one may note that the electric field, $F_{t r}^{\prime}$ which is proportional to $S^{\prime}$ should be zero as $r$ goes to infinity. We employ a grid of points $r_{i}$, where $r_{i}$ goes from $r_{H}$ to some large value of $r\left(r_{\infty}\right)$ which is much greater than $r_{H}$. We rewrite Eqs. (15)-(17) in a finite deference language and use the over relaxation method 25] to calculate the numerical solutions of $X(r), P(r)$ and $S(r)$ for different values of the rotation parameter and winding number. The numerical results of calculations are shown in Figs 1,5.

First, we investigate the influence of rotation parameter on the solutions of field equations (15)-(17). We carry out all the calculation for $l=1, \lambda=0.2$ and $b=0.3$ for which the radius 


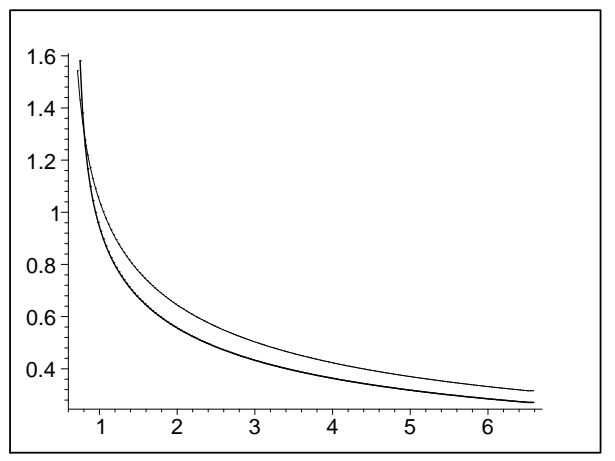

FIG. 2: $H_{\text {Higgs }}$ versus $r$ for $N=1, a=0$ (solid) and 0.7 (bold).

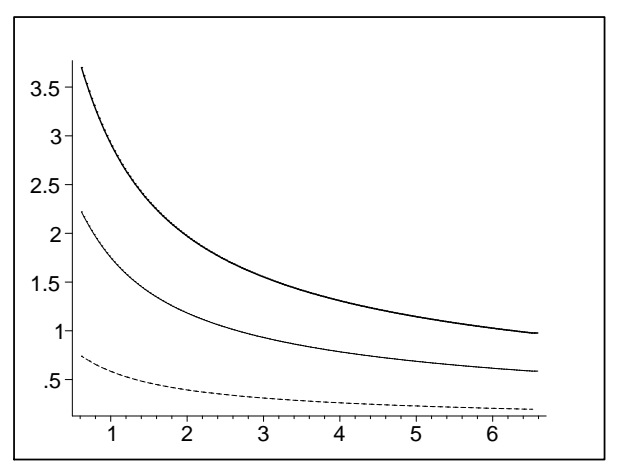

FIG. 3: $E_{\text {Higgs }} \times 10^{3}$ versus $r$ for $N=1$ (dotted), 3 (solid), and 5 (bold).

of horizon is $r_{H}=0.6173$. Figures 1 and 2 show the behavior of the electric, $E_{\text {Higgs }}=F_{t r}^{\prime}$, and the magnetic, $H_{\text {Higgs }}=F_{\phi r}^{\prime}$, fields associated with the field $P_{\mu}$ respectively for different values of the rotation parameters. We use the subscript "Higgs" for these electromagnetic field to emphasis that they are coupled with the Higgs scalar field $\Phi$. As one can see in Fig. 1. $E_{\text {Higgs }}$ is zero for $a=0$, and becomes larger as $a$ increases. The magnetic field

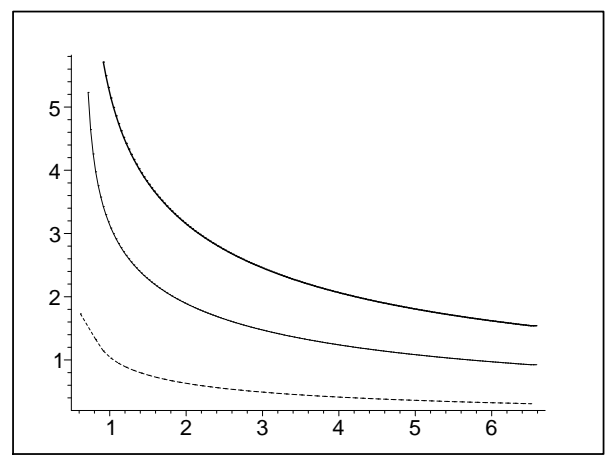

FIG. 4: $H_{\text {Higgs }}$ versus $r$ for $N=1$ (dotted), 3 (solid), and 5 (bold). 


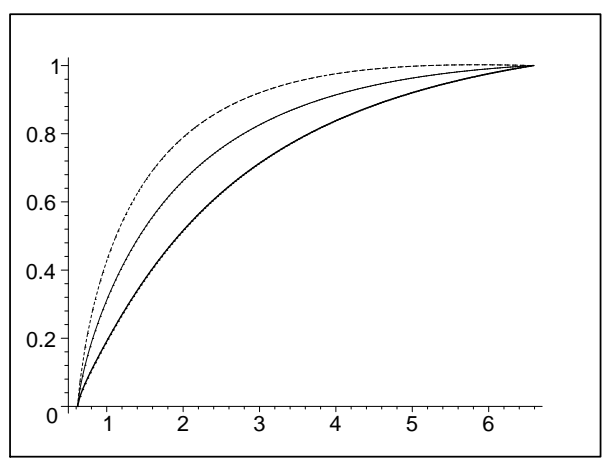

FIG. 5: $X(r)$ versus $r$ for $N=1$ (dotted), 3 (solid), and 5 (bold).

$H_{\text {Higgs }}$ is plotted in Fig. 2 for different values of angular momentum. This figure shows that the variation of $H_{\text {Higgs }}$ with respect to the rotation parameter is very slow. Overall, these figures show that the vortex thickness decreases as the rotation parameter increases. As we mentioned, in the case of non vanishing rotation parameter we encounter with the electric field $E_{\text {Higgs }}$. One may compute the source of this electric field through the use of Gauss law numerically. It is notable that the computation of this electric type charge for $E_{H i g g s}$ shows that it increases as $a$ becomes larger. This is analogous to the rotating solutions of Einstein-Maxwell equation discussed in the context of cosmic string theory for which the electric charge of the string is proportional to the rotation parameter of the string [26].

Next we investigate the influence of the winding number $N$ on the solutions of field equations (15)-(17) for the case of rotating charged black string. The results for $a=0.5$ are shown in Figs. 3, 4 and 5 for different values of $N$. As in the case of asymptotically flat, dS, and AdS spacetimes considered in Refs. [8] and [17, 18, 19, 20], increasing the winding number yields a greater vortex thickness.

The effects of charge or mass parameters on the solutions of field equations (15)-(17) for the case of rotating charged black string are the same as the nonrotating case, which is considered in [22]. The extreme black string needs more investigation. Bonjour et.al. 12] have shown that vortices of size smaller than a certain fraction of the radius of the Reissner-Nordström black hole will definitely pierce the horizon, whereas vortices thicker than a certain lower bound will instead wrap the black hole. We will consider the extreme case in future. 


\section{ASYMPTOTIC BEHAVIOR OF THE SOLUTIONS OF EINSTEIN-MAXWELL-HIGGS EQUATION}

In previous section we found the solutions of Higgs field equation in the background of charged rotating black string. Here we want to solve the coupled Einstein-Maxwell-Higgs differential equation (5)-(8). This is a formidable problem even for flat or AdS spacetimes, and no exact solutions have been found for these spacetimes yet. Indeed, besides the electromagnetic stress energy tensor, the energy-momentum tensor of the Higgs field is also a source for Einstein equation (8). However, some physical results can be obtained by making some approximations. First, we assume that the thickness of the skin covering the black string is much smaller than all the other relevant length scales. Second, we assume that the gravitational effects of the Higgs field are weak enough so that the linearized EinsteinAbelian Higgs differential equations are applicable. We choose $g_{\mu \nu} \simeq g_{\mu \nu}^{(0)}+\varepsilon g_{\mu \nu}^{(1)}$, where $g_{\mu \nu}^{(0)}$ is the rotating charged black string metric in the absence of the Higgs field and $g_{\mu \nu}^{(1)}$ is the first order correction to the metric. Employing the two assumptions concerning the thickness of the vortex and its weak gravitational field, the first order approximation to Einstein equation (8) can be written as:

$$
G_{\mu \nu}^{(1)}-\frac{3}{l^{2}} g_{\mu \nu}^{(1)}=\mathcal{T}_{\mu \nu}^{(0)}
$$

where $G_{\mu \nu}^{(1)}$ is the first order correction to the Einstein tensor due to $g_{\mu \nu}^{(1)}$ and $\mathcal{T}_{\mu \nu}^{(0)}$ is the energy-momentum tensor of the Higgs field in the rotating charged black string background metric with components:

$$
\begin{aligned}
\mathcal{T}_{t}^{t(0)}(r)= & \left\{-\Gamma l^{4}\left(r^{2} \Xi^{2}-\Gamma a^{2}\right){S^{\prime}}^{2}-\alpha r^{2} l^{4} \Gamma^{2} X^{\prime^{2}}-N^{2} \Gamma\left(\Xi^{2} l^{4} \Gamma-a^{2} r^{2}\right) P^{\prime^{2}}\right. \\
& -\alpha N^{2} X^{2} l^{4} \Gamma P^{2}-\alpha a^{2}\left(\Gamma l^{2}-r^{2}\right)\left(N^{2} P^{2}-S^{2} l^{2}\right) X^{2}-\alpha r^{2} l^{4} X^{2} S^{2} \\
& \left.-2 \alpha r^{2} l^{4} \Gamma\left(X^{2}-1\right)^{2}\right\} / 2 r^{2} l^{4} \Gamma, \\
\mathcal{T}_{\phi}^{\phi(0)}(r)= & \left\{\Gamma l^{4}\left(-\Gamma a^{2}+r^{2} \Xi^{2}\right){S^{\prime}}^{2}-\alpha r^{2} l^{4} \Gamma^{2} X^{\prime 2}+N^{2} \Gamma\left(\Gamma \Xi^{2} l^{4}-a^{2} r^{2}\right) P^{\prime 2}\right. \\
& +\alpha l^{4} X^{2}\left(N^{2} \Gamma P^{2}+r^{2} S^{2}\right)+\alpha X^{2} a^{2}\left(\Gamma l^{2}-r^{2}\right)\left(N^{2} P^{2}-l^{2} S^{2}\right) \\
& \left.-2 \alpha \Gamma r^{2} l^{4}\left(X^{2}-1\right)^{2}\right\} / 2 r^{2} l^{4} \Gamma, \\
\mathcal{T}_{\phi}^{t(0)}(r)= & \left\{2 N^{2} l^{2} \Gamma a \Xi\left(\Gamma l^{2}-r^{2}\right) P^{\prime 2}+2 \Gamma l^{4} N\left(\Gamma a^{2}-r^{2} \Xi^{2}\right) P^{\prime} S^{\prime}-r^{2} l^{2} P S\right. \\
& \left.+2 l^{2} N X^{2} \alpha\left(P a\left(\Gamma l^{2}-r^{2}\right)(a S+\Xi N P)\right)\right\} / 2 r^{2} l^{4} \Gamma, \\
\mathcal{T}_{r}^{r(0)}(r)= & \left\{\Gamma l^{4}\left(\Gamma a^{2}-\Xi^{2} r^{2}\right) S^{\prime 2}+\alpha r^{2} l^{4} \Gamma^{2} X^{\prime 2}+N^{2} \Gamma\left(\Gamma \Xi^{2} l^{4}-a^{2} r^{2}\right) P^{\prime 2}\right.
\end{aligned}
$$




$$
\begin{aligned}
& +2 a \Xi l^{2} N \Gamma\left(\Gamma l^{2}-r^{2}\right) P^{\prime} S^{\prime}-2 \alpha r^{2} l^{4} \Gamma\left(X^{2}-1\right)^{2}-\alpha X^{2} a^{2} \times \\
& \left(\Gamma l^{2}-r^{2}\right)\left(N^{2} P^{2}+l^{2} S^{2}\right)-2 \alpha l^{2} X^{2} N a \Xi S P\left(\Gamma l^{2}-r^{2}\right) \\
& \left.-\alpha l^{4} X^{2}\left(\Gamma N^{2} P^{2}-r^{2} S^{2}\right)\right\} / 2 r^{2} l^{4} \Gamma, \\
\mathcal{T}_{z}^{z(0)}(r)= & \left\{\Gamma l^{4}\left(-\Gamma a^{2}+r^{2} \Xi^{2}\right) S^{\prime 2}-\alpha r^{2} l^{4} \Gamma^{2} X^{\prime 2}-N^{2} \Gamma\left(\Gamma l^{4} \Xi^{2}-a^{2} r^{2}\right) P^{\prime 2}\right. \\
& -2 a l^{2} \Xi N \Gamma\left(\Gamma l^{2}-r^{2}\right) P^{\prime} S^{\prime}-2 \alpha r^{2} l^{4} \Gamma\left(X^{2}-1\right)^{2}-\alpha X^{2} a^{2} \times \\
& \left(\Gamma l^{2}-r^{2}\right)\left(N^{2} P^{2}+l^{2} S^{2}\right)-2 \alpha l^{2} X^{2} N a \Xi S P\left(\Gamma l^{2}-r^{2}\right) \\
& \left.-\alpha l^{4} X^{2}\left(N^{2} \Gamma P^{2}-r^{2} S^{2}\right)\right\} / 2 r^{2} l^{4} \Gamma,
\end{aligned}
$$

where $X, P$, and $S$ are the solutions of the Abelian Higgs system. As we mentioned in the last section, the vortex thickness decreases as the rotation parameter increases. This fact is more clear in Fig. [6 which shows $T_{t}^{t(0)}$ for various values of $a$.

For convenience, we use the following form, which has cylindrical symmetry, of the metric

$$
d s^{2}=-\widetilde{A}(r) d t^{2}+\widetilde{B}(r) d r^{2}+\widetilde{C}(r) d t d \phi+\widetilde{D}(r) d \phi^{2}+\widetilde{E}(r) d z^{2}
$$

In order to solve numerically Eq. (18), it is better to write the metric function $\widetilde{A}(r)$ to $\widetilde{E}(r)$ as

$$
\begin{aligned}
& \widetilde{A}(r)=A_{0}(r)[1+\varepsilon A(r)], \\
& \widetilde{B}(r)=B_{0}(r)[1+\varepsilon B(r)], \\
& \widetilde{C}(r)=C_{0}(r)[1+\varepsilon C(r)], \\
& \widetilde{D}(r)=D_{0}(r)[1+\varepsilon D(r)], \\
& \widetilde{E}(r)=E_{0}(r)[1+\varepsilon E(r)],
\end{aligned}
$$

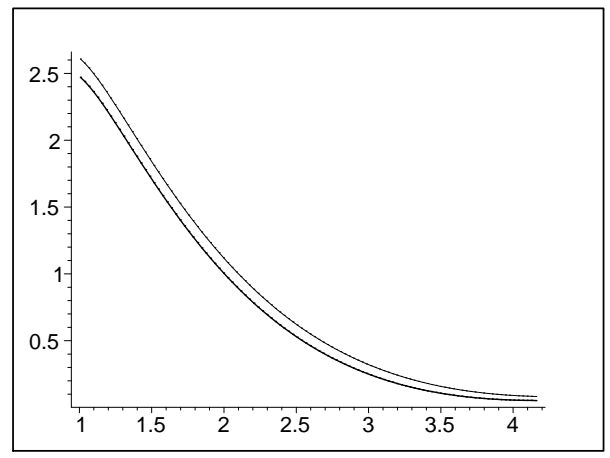

FIG. 6: $\mathcal{T}_{t}^{t(0)}$ versus $r$ for $a=0$ (solid) and $a=0.7$ (bold). 


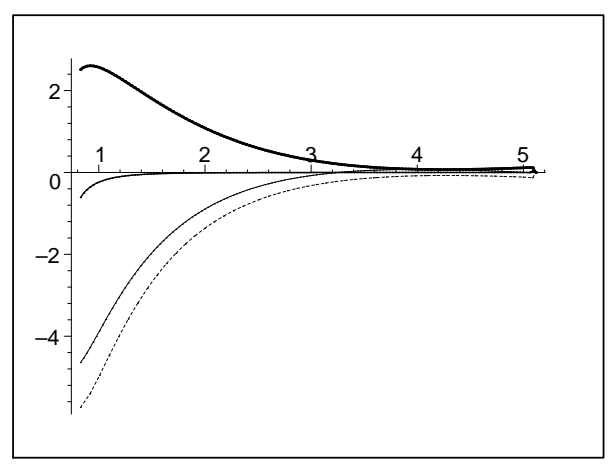

FIG. 7: $\mathcal{T}_{t}^{t(0)}=\mathcal{T}_{z}^{z(0)}$ (broken), $\mathcal{T}_{\phi}^{\phi(0)}$ (solid), $\mathcal{T}_{\phi}^{t(0)}$ (bold) and $\mathcal{T}_{r}^{r(0)}$ (thick-bold), versus $r$ for $N=1, a=0.5$

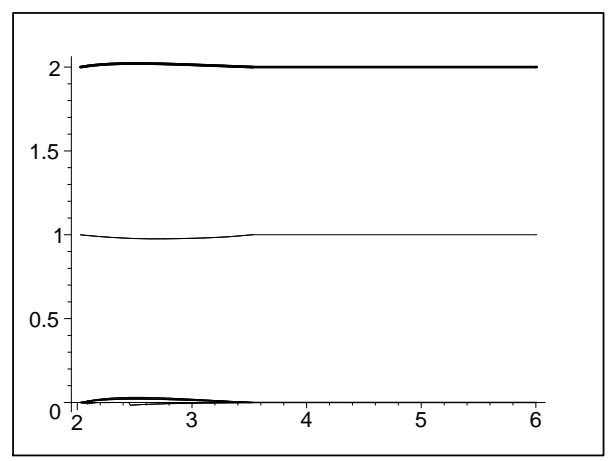

FIG. 8: $A(r), B(r)$ and $E(r)$ touch the horizontal axis, $C(r)$ (solid) and $D(r)$ (bold).

where $A_{0}(r)=\Gamma \Xi^{2}-r^{2} a^{2} l^{-4}, B_{0}(r)=\Gamma^{-1}, C_{0}=2 a \Xi\left(\Gamma-r^{2} l^{-2}\right), D_{0}(r)=r^{2} \Xi^{2}-\Gamma a^{2}$, $E_{0}(r)=r^{2} l^{-2}$, yielding the metric of the stationary rotating charged black string in four dimensions. The Einstein equations (18) in terms of the functions $A(r)$ to $E(r)$ are given in the appendix. Here we want to obtain the behavior of these functions for large values of the coordinate $r$. As one can see from Fig. 17, the components of the energy-momentum tensor rapidly go to zero outside the skin, so the situation is the same as what happened in the static black string spacetime considered in [20]. One can solve the linearized Einstein equation for large values of $r$ numerically. The results which are displayed in Fig. 8 show that $A(r)=B(r)=E(r)=0$, and $2 C(r)=D(r)=2$. Hence the metric (20) can be written as

$$
d s^{2}=-A_{0}(r) d t^{2}+B_{0}(r) d r^{2}+(1+\varepsilon) C_{0}(r) d t d \phi+(1+2 \varepsilon) D_{0}(r) d \phi^{2}+E_{0}(r) d z^{2} .
$$

It is worthwhile to mention that the metric (22) is the first order solution in $\varepsilon$ of the EinsteinMaxwell-Higgs equations far from the thin string. Of course, one may note that the metric 
(22) is the first order approximation of the following metric

$$
d s^{2}=-\Gamma(\Xi d t-a \beta d \phi)^{2}+\frac{r^{2}}{l^{4}}\left(a d t-\Xi l^{2} \beta d \phi\right)^{2}+\frac{d r^{2}}{\Gamma}+\frac{r^{2}}{l^{2}} d z^{2},
$$

which is the exact solution of Einstein-Maxwell gravity. In Eq. (23), $\beta$ is defined as $\beta=1+\varepsilon$. The above metric describes a stationary rotating charged black string with a deficit angle $\Delta=2 \pi \varepsilon$. The size of deficit angle $\Delta$ is proportional to $2 \pi \int r T_{t}^{t(0)} d r[\underline{8}]$. Numerical computation shows that the absolute values of this integral decreases as the rotation parameter increases, which can also been from Fig. 6. So, using a physical Lagrangian based model, we have established that the presence of the Higgs field induces a deficit angle in the rotating charged black string metric which decreases as the rotation parameter increases.

\section{CONCLUSION}

The effect of a vortex on pure AdS spacetime is to create a deficit angle in the metric in the thin vortex approximation. This fact has been extended to the charged rotating spacetimes. This is done by including the self-gravity of the thin vortex in the rotating charged black string background in the first order approximation discussed in Sec. IV As in the case of pure AdS [17], Schwarzschild-AdS [19], Kerr-AdS, and Reissner-NordströmAdS [21] spacetimes, we found out that the effect of a thin vortex on the stationary charged black string is to create a deficit angle in the metric which decreases as the rotation parameter increases.

We considered the Abelian Higgs field in the background of a stationary rotating charged black string. We obtained the numerical solutions for various values of rotation parameter and found that for a fixed horizon radius, by increasing the rotation parameter the vortex thickness decrease very slowly. Also the numerical solutions for various values of winding number were obtained. These solutions shows that the vortex thickness increases as winding number increases. These situations hold also in the case of the extremal black string.

The main difference between the case of Abelian Higgs field in the background of static black string considered in [20], and this paper is that the time component of the gauge field coupled to the Higgs scalar field is not zero for non zero rotation parameter. Indeed, we found that for the case of rotating black string, there exist an electric field coupled to the Higgs scalar field. This electric field increases as the rotation parameter becomes larger. 
Numerical calculations show that the electric charge which creates this electric field grows up as the rotation parameter increases. This is analogous to the results that Dias and Lemos have found recently for the magnetic rotating string [26]. They showed that the charge per unit length of a rotating string in the Einstein-Maxwell gravity increases as the rotation parameter becomes larger, and we found that the electric charge of the field $F_{\mu \nu}^{\prime}$ coupled to the Higgs field has the same feature.

Other related problems such as a study of extreme black string in the presence of Abelian Higgs field, a study of the non-Abelian vortex solution in asymptotically AdS spacetimes, or other kinds of fields coupled with gravity remain to be carried out. Work on these problems is in progress.

[1] R. Ruffini and J. A. Wheeler. Phys. Today 24, 30 (1971).

[2] D. Sudarsky, Class. Quantum Grav. 12, 579 (1995).

[3] A. Saa, J. Math. Phys. 37, 2346 (1996); A. Saa, Phys. Rev. D 53, 7377 (1996); A. E. Mayo and J. D. Bekenstein, Phys. Rev. D 54, 5059 (1996).

[4] U. Nucamendi and M. Salgado, Phys. Rev. D. 68, 04026 (2003).

[5] M. S. Volkov and D. V. Gal'tsov, Y. Fiz. 51, 1171 (1990) [Sov. J. Nucl. Phys. 51, 747 (1990)]; P. Bizon, Phys. Rev. Lett. 64, 2844 (1990); H. P. Kunzle and A. K. Masoud-ul-Alam, J. Math. Phys. 31, 928 (1990).

[6] N. Straumann and Z. H. Zhou, phys. Lett. B 243,33 (1990); Z. H. Zhou and N. Straumann, Nucl. Phys. B 360, 180 (1991); P. Bizon, Phys. Lett. B 259, 53 (1991); M. S. Volkov, et al., Phys. Lett. B 349, 438 (1995); O. Brodbeck, N. Straumann, J. Math. Phys. 37, 1414 (1996).

[7] H. Watab and T. Torii, JCAP 0402, 001 (2004).

[8] A. Achucarro, R. Gregory, and K. Kuijken, Phys. Rev. D 52, 5729 (1995).

[9] A. Chamblin, J. M. A. Ashbourn-Chamblin, R. Emparan, and A. Sornborger, Phys. Rev. D 58, 124014 (1998).

[10] A. Chamblin, J. M. A. Ashbourn-Chamblin, R. Emparan, and A. Sornborger, Phys. Rev. Lett. 80, 4378 (1998).

[11] F. Bonjour and R. Gregory, Phys. Rev. Lett. 81, 5034 (1998). 
[12] F. Bonjour, R. Emparan, and R. Gregory, Phys. Rev. D. 59, 84022 (1999).

[13] E. Winstanley, Found. Phys. 33, 111 (2002).

[14] T. Torii, K. Maeda, and M. Narita, Phys. Rev. D 64, 042116 (2001).

[15] E. Winstanley, Class. Quantum Grav. 16, 1963 (1999).

[16] H. B. Nielsen and P. Olesen, Nucl. Phys. B 61, 45 (1973).

[17] M. H. Dehghani, A. M. Ghezelbash, and R. B. Mann, Nucl. Phys. B 625, 389 (2002).

[18] A. M. Ghezelbash and R. B. Mann, Phys. Lett B 537, 329 (2002).

[19] M. H. Dehghani, A. M. Ghezelbash, and R. B. Mann, Phys. Rev. D 65, 044010 (2002).

[20] M. H. Dehghani and T. Jalali, Phys. Rev. D 66, 124014 (2002).

[21] A. M. Ghezelbash and R. B. Mann, Phys. Rev. D 65, 124022 (2002).

[22] M. H. Dehghani, Phys. Rev. D 66, 044006 (2002).

[23] M. H. Dehghani, A. Khodam-Mohammadi, Phys. Rev. D 67, 084006 (2003).

[24] J. P. S. Lemos and V. T. Zanchin, Phys. Rev. D 54, 3840 (1996).

[25] W. H. Press, S. A. Teukolsky, W. T. Vetterling and B. P. Flannery, Numerical Recipes in FORTRAN (Cambridge University Press, Cambridge, England, 1992).

[26] O. J. C. Dias and J. P. S. Lemos, Class. Quantum Grav. 19, 2265 (2002).

\section{APPENDIX}

The Einstein equation mentioned in Sec. (IV) in terms of the metric functions $A(r)$ to $E(r)$ are

$$
\begin{aligned}
& \Xi^{2} \digamma_{1}(A+D-2 C)-4 \lambda^{2} l^{4} a^{2} \Gamma^{2}\left(\Gamma \Xi^{2} l^{4}-r^{2} a^{2}\right) D+4 \lambda^{2} l^{8} \Xi^{2} \Gamma^{2}\left(\Gamma a^{2}-r^{2} \Xi^{2}\right) A-r^{4} l^{2} \Gamma^{2} \times \\
& \left\{4 r l^{4} \Xi^{2} \Gamma+\left(b l^{3}-4 r^{3} a^{2}\right)\right\} B^{\prime}-\Xi^{2} a^{2} l^{8} r \Gamma^{2}\left\{2 b^{2} r^{2}+\lambda^{2}\left(4 \lambda^{2} l^{2}-6 b l r\right)\right\} A^{\prime}-12 l^{4} r^{6} \Gamma^{2} B \\
& -\Xi^{2} r^{2} l^{2} \Gamma \digamma_{2}\left(A^{\prime}+D^{\prime}-2 C^{\prime}\right)+l^{2} \Gamma^{2} r^{4}\left\{4 r l^{4} \Gamma \Xi^{2}+\left(4 r^{3}-b l^{3}\right)\left(a^{2}-l^{2}\right)\right\}\left(E^{\prime}+D^{\prime}\right) \\
& +2 \Gamma^{3} r^{6} l^{6}\left(E^{\prime \prime}+D^{\prime \prime}\right)+\Gamma^{2} l^{4} a^{2}\left\{2 \Xi^{2} l^{4}\left[b^{2} r+\lambda^{2}\left(2 \lambda^{2} l^{2} r^{-1}-3 b l\right)\right]+r^{3}\left(4 \lambda^{2} l^{2}-3 b l r\right)\right\} D^{\prime} \\
& -2 \Xi^{2} a^{2} \lambda^{2} l^{6} r \Gamma^{2}\left(\Gamma r l^{2}-r^{3}-b l^{3}\right)\left(D^{\prime \prime}-C^{\prime \prime}\right)=4 r^{6} l^{6} \Gamma^{2} \mathcal{T}_{t}^{t(0)}, \\
& 8 \Gamma^{2}\left(\Gamma l^{2}-r^{2}\right) \lambda^{2} \Xi l^{6} a^{2} D+8 \lambda^{2} \Xi l^{6} \Gamma^{2} a\left\{l^{2} r^{2}-\left(\Gamma l^{2}-r^{2}\right) a^{2}\right\} C+a \Xi^{3} \digamma_{3}(A+D-2 C)+ \\
& r^{4} l^{4} a \Xi \Gamma^{2}\left(4 r l^{2} \Gamma-4 r^{3}+b l^{3}\right)\left(B^{\prime}-E^{\prime}-2 D^{\prime}\right)-r^{2} l^{2} a \Xi \Gamma \digamma_{5}\left(A^{\prime}-D^{\prime}\right)-2 r^{2} l^{2} \Xi a \Gamma \digamma_{4} \times \\
& \left(C^{\prime}-D^{\prime}\right)+2 l^{4} \Gamma^{2} \Xi a\left\{l^{5} r^{2}\left(\Xi^{2}-1\right) \Gamma\left(\lambda^{2} l-b r\right)+\Xi^{2} r^{6}\left(r^{2}-\Gamma l^{2}\right)\right\}\left(D^{\prime \prime}-C^{\prime \prime}\right)=4 r^{6} l^{6} \Gamma^{2} \mathcal{T}_{\varphi}^{t(0)}, \\
& \digamma_{6}(A+D-2 C)+8 a^{2} \lambda^{2} l^{6} \Gamma^{2}\left\{\Gamma l^{2} \Xi^{2}-r^{2}\left(-1+\Xi^{2}\right)\right\}(C-A)+4 r^{2} l^{6} \lambda^{2} \Gamma^{2}\left(\Xi^{2} l^{2}+a^{2}\right) A+
\end{aligned}
$$




$$
\begin{aligned}
& r^{2} \Gamma l^{-4} \Xi^{2} \digamma_{7}\left(A^{\prime}+D^{\prime}-2 C^{\prime}\right)-4 \Xi^{2} a^{2} l^{6} r \Gamma^{2}\left\{b^{2} r l^{2}+\lambda^{2}\left(2 \Gamma r l^{2}-b l^{3}-2 r^{3}\right)\right\} C^{\prime}+l^{4} r^{2} \Gamma^{2} \digamma_{8} A^{\prime} \\
& +r^{4} l^{4} \Gamma^{2}\left(4 r^{3}-b l^{3}\right) E^{\prime}+r^{4} l^{4} \Gamma^{2}\left\{4 r a^{2} \Gamma-\Xi^{2}\left(4 r^{3}-b l^{3}\right)\right\}\left(B^{\prime}-E^{\prime}\right)+2 r^{6} l^{6} \Gamma^{3}\left(E^{\prime \prime}+A^{\prime \prime}\right) \\
& -2 \Xi^{2} a^{2} \lambda^{2} l^{6} r \Gamma^{2}\left(\Gamma l^{2} r-r^{3}-b l^{3}\right)\left(A^{\prime \prime}-C^{\prime \prime}\right)-12 l^{4} r^{6} \Gamma^{2} B=4 r^{6} l^{6} \Gamma^{2} \mathcal{T}_{\varphi}^{\varphi(0)}, \\
& \Xi^{2} \digamma_{9}(A+D-2 C)-8 r^{2} \lambda^{2} \Xi^{2} l^{6} \Gamma C+4 r^{2} \lambda^{2} l^{4} \Gamma\left(\Xi^{2} l^{2}-a^{2}\right) D+r^{4} l^{2} \Gamma\left(4 r^{3}-b l^{3}\right) E^{\prime}+r^{4} l^{2} \Gamma \\
& \times\left\{4 r a^{2} \Gamma-\Xi^{2}\left(4 r^{3}-b l^{3}\right)\right\} D^{\prime}+r^{4} \Gamma\left\{4 r l^{4} \Xi^{2} \Gamma-a^{2}\left(4 r^{3}-b l^{3}\right)\right\} A^{\prime}+\Xi^{2} a^{2} b l^{3} r^{2} \Gamma\left(\Gamma l^{2}-r^{2}\right) \\
& \times\left(A^{\prime}+D^{\prime}-2 C^{\prime}\right)-12 r^{6} l^{2} \Gamma B=4 r^{6} l^{4} \Gamma \mathcal{T}_{r}^{r(0)}, \\
& \Xi^{2} \digamma_{10}(A+D-2 C)-16 r^{2} \lambda^{2} l^{6} \Gamma^{2}\left(\Xi^{2} l^{2}-a^{2}\right) D+\left(32 \lambda^{2} \Xi^{2} l^{8} r^{2} \Gamma^{2}\right) C-4 l^{4} r^{4} \Gamma^{2}\left(4 r^{3}-b l^{3}\right) B^{\prime}+ \\
& 4 l^{4} r^{2} \Gamma^{2}\left\{8 r^{3} l^{2} \Gamma+\left(4 \lambda^{2} l^{2}-3 b l r\right)\left(a^{2}-2 l^{2}\right)\right\} D^{\prime}+4 l^{4} r^{3} \Gamma^{2}\left\{8 r^{4}+\left(3 \Xi^{2}-2\right) b l^{2} r-4 \lambda^{2} l^{4} \Xi^{2}\right\} A^{\prime} \\
& +4 l^{2} r^{2} \Gamma \Xi^{2} \digamma_{11}\left(A^{\prime}+D^{\prime}-2 C^{\prime}\right)-8 \Xi^{2} a^{2} \lambda^{2} l^{6} r \Gamma^{2}\left(r l^{2} \Gamma-r^{3}-b l^{3}\right)\left(A^{\prime \prime}+D^{\prime \prime}-2 C^{\prime \prime}\right)+8 r^{6} l^{6} \Gamma^{3} \\
& \times\left(A^{\prime \prime}+D^{\prime \prime}\right)-48 l^{4} r^{6} \Gamma^{2} B=16 r^{6} l^{6} \Gamma^{2} \mathcal{T}_{z}^{z(0)},
\end{aligned}
$$

where $\mathcal{T}_{\mu}^{\nu(0)}$ is the energy momentum tensor of the Higgs field given in Eqs. (19) and the functions $\digamma_{i}$ 's are:

$$
\begin{aligned}
& \digamma_{1}=\Xi^{4}\left(32 \Gamma^{3} l^{6} r^{4}-\Gamma^{2} l^{10} b^{2}+r^{4} b^{2} l^{6}-32 \Gamma l^{2} r^{8}-8 \Gamma^{3} l^{9} r b+8 \Gamma^{2} l^{7} r^{3} b+16 r^{10}-16 \Gamma^{4} l^{8} r^{2}-8 r^{7} b l^{3}\right. \\
& \left.+8 \Gamma l^{5} r^{5} b\right)+\Xi^{2}\left(\Gamma^{2} l^{10} b^{2}+12 \Gamma^{4} l^{8} r^{2}-28 \Gamma^{3} l^{6} r^{4}-12 \Gamma^{2} l^{4} r^{6}+60 \Gamma l^{2} r^{8}-32 r^{10}+16 r^{7} b l^{3}\right. \\
& \left.-12 \Gamma l^{5} r^{5} b+4 \Gamma^{3} l^{9} r b-8 \Gamma^{2} l^{7} r^{3} b-2 r^{4} b^{2} l^{6}\right)+4 \Gamma^{4} l^{8} r^{2}+r^{4} b^{2} l^{6}-8 r^{7} b l^{3}+4 \Gamma^{3} l^{9} r b+16 r^{10} \\
& +4 \Gamma l^{5} r^{5} b+12 \Gamma^{2} l^{4} r^{6}-28 \Gamma l^{2} r^{8}-4 \Gamma^{3} l^{6} r^{4} \\
& \digamma_{2}=\Xi^{4}\left(4 r^{7}-4 \Gamma^{3} l^{6} r-b l^{3} r^{4}-b l^{7} \Gamma^{2}+2 b l^{5} \Gamma r^{2}+12 \Gamma^{2} l^{4} r^{3}-12 \Gamma l^{2} r^{5}\right)+\Xi^{2}\left(2 r^{4} b l^{3}-8 r^{7}-b l^{7} \Gamma^{2}\right. \\
& \left.+16 r^{5} \Gamma l^{2}-8 r^{3} \Gamma^{2} l^{4}-r^{2} b l^{5} \Gamma\right)-r^{4} b l^{3}+4 r^{7}+2 \Gamma^{2} l^{7} b-\Gamma l^{5} r^{2} b+4 \Gamma^{3} l^{6} r-4 \Gamma^{2} l^{4} r^{3}-4 \Gamma l^{2} r^{5}, \\
& \digamma_{3}=\Xi^{2}\left(32 \Gamma l^{2} r^{8}-32 \Gamma^{3} l^{6} r^{4}+8 r^{7} b l^{3}-r^{4} b^{2} l^{6}-8 \Gamma^{2} l^{7} r^{3} b-8 \Gamma l^{5} r^{5} b+8 \Gamma^{3} l^{9} r b+\Gamma^{2} l^{10} b^{2}-\right. \\
& \left.16 r^{10}+16 \Gamma^{4} l^{8} r^{2}\right)+8 \Gamma l^{5} r^{5} b+16 r^{10}+32 \Gamma^{3} l^{6} r^{4}+8 \Gamma^{2} l^{7} r^{3} b-8 \Gamma^{3} l^{9} r b-16 \Gamma^{4} l^{8} r^{2}-32 \Gamma l^{2} r^{8} \\
& +r^{4} b^{2} l^{6}-8 r^{7} b l^{3}-b^{2} l^{10} \Gamma^{2}, \\
& \digamma_{4}=\Xi^{4}\left(4 r^{7}-b l^{7} \Gamma^{2}-b l^{3} r^{4}+12 \Gamma^{2} l^{4} r^{3}-12 \Gamma l^{2} r^{5}-4 \Gamma^{3} l^{6} r+2 b l^{5} \Gamma r^{2}\right)+\Xi^{2}\left(8 r^{5} \Gamma l^{2}-b l^{7} \Gamma^{2}\right. \\
& \left.-4 r^{7}-4 r^{3} \Gamma^{2} l^{4}+r^{4} b l^{3}\right)+2 \Gamma^{2} l^{7} b+4 \Gamma^{3} l^{6} r-4 \Gamma^{2} l^{4} r^{3}, \\
& \digamma_{5}=\Xi^{4}\left(4 \Gamma^{3} l^{6} r-4 r^{7}+b l^{7} \Gamma^{2}-2 b l^{5} \Gamma r^{2}-12 \Gamma^{2} l^{4} r^{3}+12 \Gamma l^{2} r^{5}+b l^{3} r^{4}\right)+\Xi^{2}\left(2 r^{2} b l^{5} \Gamma-b l^{7} \Gamma^{2}\right. \\
& \left.+4 r^{7}+12 r^{3} \Gamma^{2} l^{4}-12 r^{5} \Gamma l^{2}-4 \Gamma^{3} l^{6} r-r^{4} b l^{3}\right)-\Gamma l^{5} r^{2} b-4 \Gamma^{2} l^{4} r^{3}+4 \Gamma l^{2} r^{5}, \\
& \digamma_{6}=\Xi^{6}\left(b^{2} l^{10} \Gamma^{2}+16 \Gamma^{4} l^{8} r^{2}-32 r^{4} \Gamma^{3} l^{6}+32 r^{8} \Gamma l^{2}+8 r^{7} b l^{3}+8 \Gamma^{3} l^{9} r b-b^{2} l^{6} r^{4}-8 r^{5} \Gamma l^{5} b-16 r^{10}\right.
\end{aligned}
$$




$$
\begin{aligned}
& \left.-8 r^{3} \Gamma^{2} l^{7} b\right)+\Xi^{4} r^{4} b^{2} l^{6}+2 \Xi^{4}\left(4 b l^{3} r^{7}-18 r^{8} \Gamma l^{2}+6 b l^{7} \Gamma^{2} r^{3}-8 b l^{9} \Gamma^{3} r+30 r^{4} \Gamma^{3} l^{6}+6 b l^{5} \Gamma r^{5}\right. \\
& \left.-4 r^{6} \Gamma^{2} l^{4}+8 r^{10}-b^{2} l^{10} \Gamma^{2}+16 \Gamma^{4} l^{8} r^{2}\right)+4 \Xi^{2}\left(r^{6} \Gamma^{2} l^{4}-6 r^{4} \Gamma^{3} l^{6}+r^{8} \Gamma l^{2}-r^{5} \Gamma l^{5} b+2 \Gamma^{3} l^{9} r b\right. \\
& \left.+4 \Gamma^{4} l^{8} r^{2}+\Gamma^{2} l^{10} b^{2} / 4\right)+4\left(\Gamma^{2} l^{4} r^{6}-\Gamma^{3} l^{6} r^{4}-\Gamma^{2} l^{7} r^{3} b\right), \\
& \digamma_{7}=\Xi^{4}\left(11 r b l^{5} \lambda^{4}-10 r^{2} b^{2} l^{4} \lambda^{2}-4 \lambda^{6} l^{6}+3 r^{3} b^{3} l^{3}\right)+\Xi^{2}\left(3 r^{6} b^{2}-7 r^{5} b l \lambda^{2}-6 r^{3} b^{3} l^{3}-22 r b l^{5} \lambda^{4}\right. \\
& \left.+8 \lambda^{6} l^{6}+4 r^{4} \lambda^{4} l^{2}+20 r^{2} b^{2} l^{4} \lambda^{2}\right)-3 r^{6} b^{2}+7 r^{5} b l \lambda^{2}-4 r^{4} \lambda^{4} l^{2}+11 r b l^{5} \lambda^{4}+3 r^{3} b^{3} l^{3}-4 \lambda^{6} l^{6} \\
& -10 r^{2} b^{2} l^{4} \lambda^{2} \\
& \digamma_{8}=4 \Gamma l^{2} r^{3}+2 l^{3} r^{2} \Xi^{2} b+8 r^{3} \Xi^{2} \Gamma l^{2}-4 l^{3} \Xi^{4} b r^{2}+8 \Xi^{4} r^{5}+4 l^{5} \Xi^{4} b \Gamma-4 l^{5} \Xi^{2} b \Gamma+8 \Xi^{4} \Gamma^{2} l^{4} r- \\
& 16 \Xi^{4} \Gamma l^{2} r^{3}-8 \Xi^{2} \Gamma^{2} l^{4} r+4 r^{5}-r^{2} b l^{3}, \\
& \digamma_{9}=\Xi^{2}\left(12 r^{4} \Gamma^{2} l^{4}-24 r^{6} \Gamma l^{2}-4 l^{7} b \Gamma^{2} r-l^{8} b^{2} \Gamma+4 r^{3} b l^{5} \Gamma+12 r^{8}\right)-16 r^{4} \Gamma^{2} l^{4}+28 r^{6} \Gamma l^{2}+b^{2} l^{8} \Gamma \\
& -8 r^{3} \Gamma l^{5} b+4 b l^{7} \Gamma^{2} r-12 r^{8}, \\
& \digamma_{10}=24 \Gamma^{3} l^{9} r b-16 \Gamma^{2} l^{7} r^{3} b+\Xi^{2}\left(76 \Gamma^{3} l^{6} r^{4}-40 \Gamma^{4} l^{8} r^{2}+20 \Gamma^{2} l^{7} r^{3} b-48 \Gamma^{2} l^{4} r^{6}+28 \Gamma l^{2} r^{8}-16 r^{10}\right. \\
& \left.-24 \Gamma^{3} l^{9} r b-2 \Gamma^{2} l^{10} b^{2}-4 \Gamma l^{5} r^{5} b-r^{4} b^{2} l^{6}+8 r^{7} b l^{3}\right)+40 \Gamma^{4} l^{8} r^{2}-72 \Gamma^{3} l^{6} r^{4}+44 \Gamma^{2} l^{4} r^{6}+ \\
& 16 r^{10}+4 \Gamma l^{5} r^{5} b-28 \Gamma l^{2} r^{8}+r^{4} b^{2} l^{6}-8 r^{7} b l^{3}+2 b^{2} l^{10} \Gamma^{2}, \\
& \digamma_{11}=\Xi^{2}\left(4 r^{7}-r^{4} b l^{3}-28 r^{3} \Gamma^{2} l^{4}-5 r^{2} b l^{5} \Gamma+6 b l^{7} \Gamma^{2}+8 r^{5} \Gamma l^{2}+16 \Gamma^{3} l^{6} r\right)+r^{4} b l^{3}-8 \Gamma l^{2} r^{5}+ \\
& 5 \Gamma l^{5} r^{2} b-4 r^{7}-6 \Gamma^{2} l^{7} b+28 \Gamma^{2} l^{4} r^{3}-16 \Gamma^{3} l^{6} r .
\end{aligned}
$$

